pledget-supported suture. We did not use this method because of suspected infection and the severely damaged PA wall. The histologic findings also support our decision. However, the PA wall was extensively abnormal, and the suture line was disrupted. Resuturing incorporating the pulmonary valve annulus caused mild residual stenosis that could not be tolerated in the presence of severe right ventricular dysfunction. Pulmonary root replacement can be a method of choice in such patients. Therefore, we believe that a pulmonary allograft should also be made available whenever possible and should be implanted during the initial operation, if necessary.

The last problem was the timing of the operation. Emergency intervention was required in most reported patients with aortopulmonary fistulae and in this patient also, although the history of the present illness was rather long, and his general condition was fair at first. His severely impaired general condition 6 days later inevitably hampered postoperative recovery. Clearly, the timing of the operation should be decided on a case-by-case basis, but we recommend performing the operation as soon as possible once the diagnosis of aortopulmonary fistula is established.

\section{References}

1. Lindsay J Jr. Aortocameral fistula: a rare complication of aortic dissection. Am Heart J. 1993;126:441-3.

2. Piciche M, De Paulis R, Chiariello L. A review of aortopulmonary fistulas in aortic dissection. Ann Thorac Surg. 1999;68:1833-6.

3. Leborgne L, Trojette F, Jarry G, Touati G, Otmani A, Hermida JS, et al. Dissection of the aorta complicated by aorto-pulmonary fistula. Arch Mal Coeur Vaiss. 2001;94:743-6.

\title{
Stent graft treatment for abdominal pseudoaneurysm near the celiac artery
}

Shoichi Takahashi, MD, ${ }^{a}$ Shunichi Takaya, MD, ${ }^{a}$ Ikuo Fukuda, MD, ${ }^{\text {a }}$ Takemichi Suto, MD, ${ }^{a}$ Kazuyuki Daitoku, MD, ${ }^{\text {a }}$ Toshihiko Kuga, MD, ${ }^{a}$ Ikko Ichinoseki, MD, ${ }^{a}$ Mamoru Munakata, MD, ${ }^{a}$ Kozo Fukui, MD, ${ }^{a}$ Hiroshi Noda, MD, and Hiraku Yodono, MD, Aomori, Japan

$\mathrm{T}$ he patient was a 56-year-old man who had a history of abdominoperineal resection and colostomy for rectal cancer 10 years before. There was no other major medical history or trauma. He was admitted to the hospital because of fever and abdominal pain that continued for 1 month. $\mathrm{He}$ was given a diagnosis of inguinal lymphadenitis and received intravenous infusion of antibiotics. Temporarily, the fever subsided, and abdominal symptoms improved. However, a high fever soon developed, and the abdominal pain returned. The patient was transferred to our hospital. Computed tomographic scanning of the chest and abdomen revealed an aneurysm of $25 \mathrm{~mm}$ in diameter. It was located on the opposite site of the celiac axis, and there was partial calcification of the aortic wall (Figure 1). Considering the clinical context and morphology of the aneurysm, a diagnosis of mycotic aneurysm was made. Administration of antibiotics and intensive antihypertensive therapy were continued until resolution

\footnotetext{
From the Departments of Surgery ${ }^{\mathrm{a}}$ and Radiology, ${ }^{\mathrm{b}}$ Hirosaki University School of Medicine, Aomori, Japan.

Received for publication Dec 2, 2002; accepted for publication Dec 16, 2002.

Address for reprints: Shoichi Takahashi, MD, Department of Cardiac Surgery, Southern Tohoku General Hospital, 7-115 Yatsuyamada, Koriyama, Fukushima 963-8563, Japan (E-mail: s.takahashi@mt.strins.or.jp).

J Thorac Cardiovasc Surg 2003;126:600-2

Copyright $\odot 2003$ by The American Association for Thoracic Surgery $0022-5223 / 2003 \$ 30.00+0$

doi:10.1016/S0022-5223(03)00127-2
}

of fever and improvement of the inflammatory marker. The fever had subsided almost completely by 1 week after transfer. The leukocyte count was $4800 / \mathrm{mm}^{3}$, and C-reactive protein levels were negative by 6 weeks after transfer. The results of blood culture, repeated several times, were negative. Because the pseudoaneurysm had been growing rapidly (computed tomography, $35 \mathrm{~mm}$ in diameter), surgical intervention was recommended. Because of the colostomy, open surgical intervention was anticipated to have high mortality and morbidity, including graft infection. Therefore stent grafting was conducted.

The patient was operated on under general anesthesia. The right femoral artery was surgically exposed. After systemic heparinization, the femoral artery was incised, and a sheath was inserted. A handmade, half-fenestrated stent graft was prepared. We used a stainless-steel Gianturco Z stent (Cook Inc, Bloomington, Ind) 30 $\mathrm{mm}$ in diameter and $50 \mathrm{~mm}$ in length. To determine its orientation, we put radiolucent markers on 2 of the 6 apexes of the stent where the graft would cover the orifice of the pseudoaneurysm (Figure 2, $B$ ) One third of the circumference of the lower three quarters of a woven graft (Ube Inc, Ube, Japan) was cut to preserve the abdominal branches, as shown in the Figure 2, A. A fan-shaped opening was also made to preserve the thick lumbar arteries. The stent was deployed with the markers lined up when viewed from the anterior perspective to preserve the branch.

The angiogram performed after deployment confirmed that the aneurysm had been excluded and that the abdominal branches were patent.

The patient was discharged without any serious complication. No signs of infection were observed for longer than 10 months after the operation. 

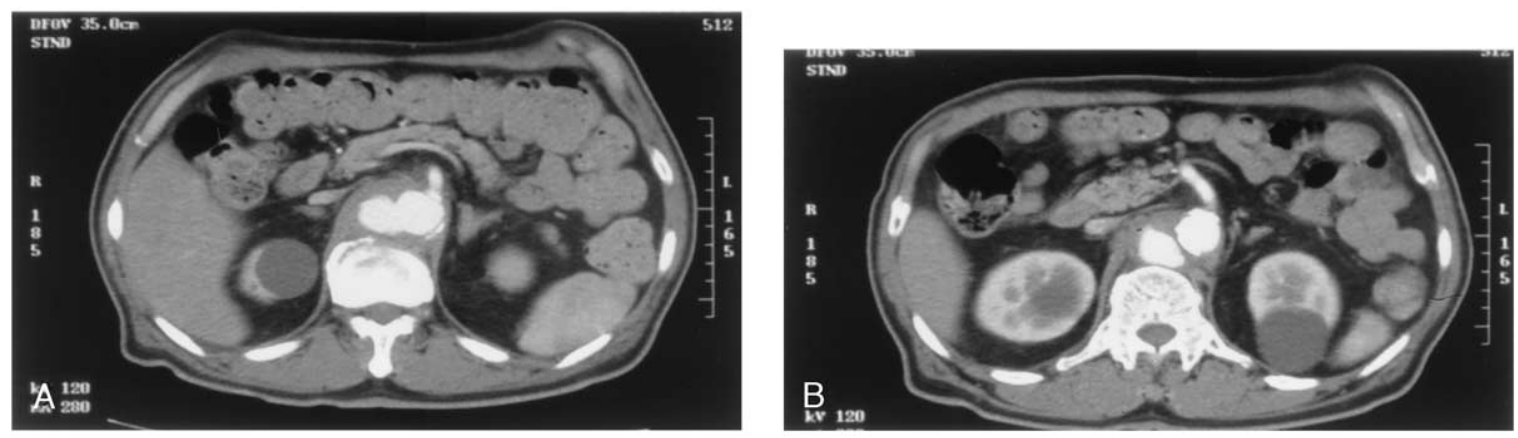

Figure 1. A, Image level with the celiac artery. B, Image level with the superior mesenteric artery. A pseudoaneurysm on the right side of the aorta was observed level with the celiac artery.
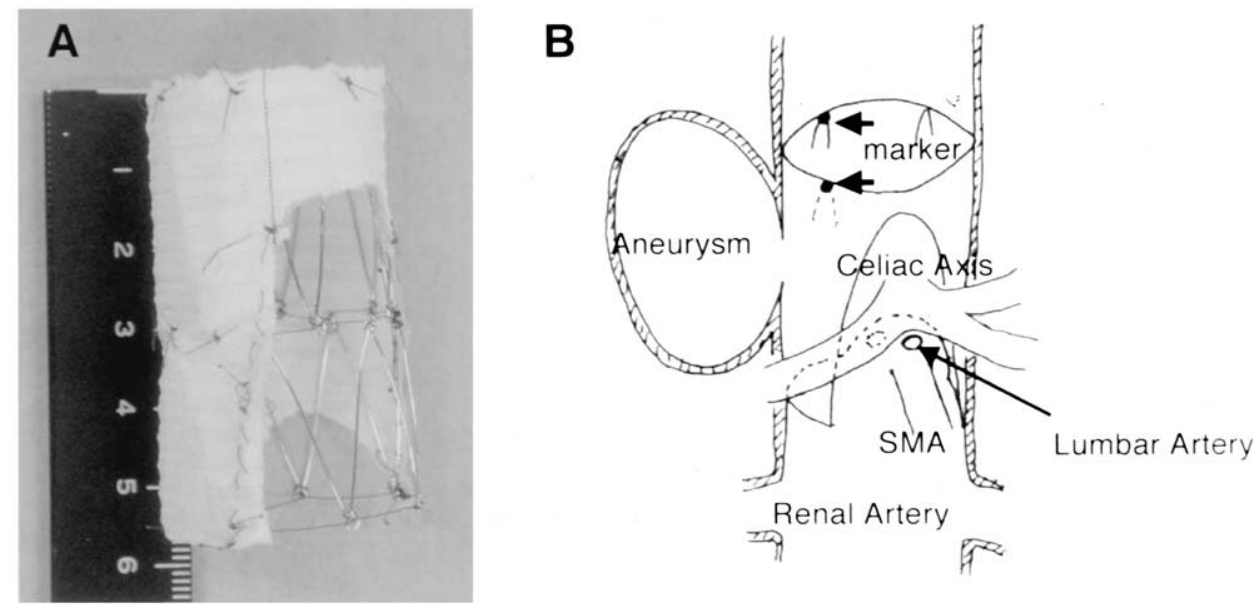

Figure 2. A, Stent covered with a partially cut synthetic graft. B, Illustration of the stent deployed to isolate the aneurysm level with the celiac artery and the superior mesenteric artery. There were markers at 2 points on the stent. SMA, Superior mesenteric artery.

\section{Discussion}

A pseudoaneurysm located at the level of the celiac artery is rare, and surgical treatment involves considerable risk. The most popular approach to this area is a retroperitoneal thoracoabdominal exposure by means of a spiral incision. However, the patient had a colostomy on the left flank, and this procedure might have damaged the colostomy or caused wound contamination, resulting in graft infection. Also, surgical exposure of the thoracoabdominal junction would be poor because of fixation of the descending colon to the abdominal wall.

Although blood culture results had been negative during the clinical course, the most probable cause of the aneurysm in this patient was bacterial infection. We presumed that even though it was a mycotic aneurysm, infection had been eradicated by antibiotic therapy. None of the symptoms suggesting systemic inflammatory response syndrome, ie, fever, increased breathing, deterioration in the general state, and laboratory test parameters, were observed when we conducted surgical intervention. Ihaya and colleagues $^{1}$ reported that graft replacement of the mycotic aneu- rysm was feasible without an increase in the incidence of graft infection when inflammatory parameters were fully controlled. Therefore we judged it would be safe to perform surgical intervention.

We chose to perform endovascular surgery to keep the surgical field free of bacterial contamination. An autologous vein-covered stent used in the treatment of mycotic aneurysm in splanchnic arteries $^{2,3}$ would reduce the risk of infection compared with use of a woven graft-covered stent. ${ }^{4,5}$ However, an autologous vein covered-stent would not applicable in this case because of the size mismatch. The stent covered with a Dacron graft was reshaped to fit the complex anatomic structure of the aorta at the thoracoabdominal junction. Kenny and colleagues ${ }^{5}$ reported efficacy of the fenestrated stent grafting in mycotic aneurysm. We used a halffenestrated stent graft to spare the visceral branch and lumbar arteries. Because the graft had spatial orientation, the rotation of the graft would induce failure, requiring urgent surgical intervention. The stent was marked to identify the spatial orientation to resolve this problem. 
In conclusion, half-fenestrated stent grafting was feasible and effective as a less invasive treatment for mycotic aneurysm when infection was medically controlled. Visceral blood supply was spared by using the technique we applied.

\section{References}

1. Ihaya A, Chiba Y, Kimura T, Morioka K, Uesaka T. Surgical outcome of infectious aneurysm of the abdominal aorta with or without SIRS. Cardiovasc Surg. 2001;9:436-40.
2. Madhavn P, McDonnell CO, Dowd MO, Sultan SAH, Doyle M, Colgan MP, et al. Suprarenal mycotic aneurysm exclusion using a stent with a partial autologous covering. J Endovasc Ther. 2000;7:404-9.

3. McGraw JK, Patzik SB, Gale SS, Dodd JT, Boorstein JM. Autogenous vein-covered stent for the endovascular management of a superior mesenteric artery pseudoaneurysm. J Vasc Interv Radiol. 1998;9:779-82.

4. Cowan S, Kahn MB, Bonn J, Becker GJ, DiMuzio P, Leichter R, et al. Superior mesenteric artery pseudoaneurysm successfully treated with polytetrafluoroethylene covered stent. J Vasc Surg. 2002;35: 805-7.

5. Kinney EV, Kaebnick HW, Mitchell RA, Jung MT. Repair of mycotic paravisceral aneurysm with a fenestrated stent-graft. J Endovasc Ther. 2000;7:192-7.

\title{
The gut-first approach for repair of Crawford extent III thoracoabdominal aortic aneurysms
}

\author{
Matthias Karck, MD, Christian Hagl, MD, Klaus Kallenbach, MD, and Axel Haverich, MD, Hannover, Germany
}

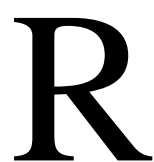

eplacement of thoracoabdominal aortic aneurysms requires reattachment of the visceral and renal artery origins to one or more side openings in the vascular graft. ${ }^{1,2}$ In Crawford extent III and IV aneurysms reimplantation and restoration of blood flow through the intestinal and renal arteries is followed by replacement of the infrarenal axis down to the aortic bifurcation or even below, with a crossclamp on the vascular graft. We describe a modification of this classical approach by interpositioning a vascular graft between the thoracic aorta and an isolated tissue patch containing the origins of the celiac, mesenteric, and left renal arteries in an end-to-end fashion. In a second step repair of the downstream aorta can be accomplished in a crossclamp-free operating field.

\section{Clinical Summary}

A 68-year-old man presented with a Crawford extent III aneurysm and chronic aortic dissection type B (Figure 1). Access to the thoracoabdominal aorta was provided by a thoracotomy in the seventh interspace, transection of the costal margin, circular division of the diaphragm, and subsequent retroperitoneal preparation. After systemic heparinization, femorofemoral cardiopulmonary bypass was initiated for maintenance of distal perfusion and in-

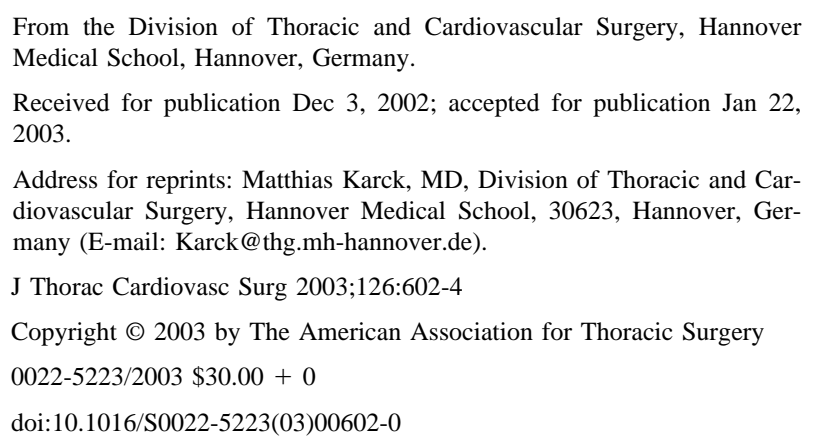

duction of mild hypothermia at $34^{\circ} \mathrm{C}$. The thoracic aorta was transected between occlusion clamps placed at the level of T4 and T7. The dissected layers of the aortic wall were reconstructed with gelatin resorcin formaldehyde glue before an anastomosis was carried out between a collagen-impregnated woven vascular graft of $32 \mathrm{~mm}$ in diameter (Hemashield, Boston Scientific) and the proximal descending aorta. Then the distal clamp was moved down to the level of the diaphragm before the aorta was incised longitudinally. Two intercostal arteries arising from T8 and T9 were reattached to the graft and reperfused after translocation of the proximal clamp. With release of the distal clamp, extracorporeal perfusion was discontinued. The aneurysm was opened longitudinally posterior to the left renal artery to its distal termination at the aortic bifurcation. The septum separating the true and false lumens was resected. The nondissected visceral and left renal artery origins were excised from the aortic wall as a single tissue patch and anastomosed end to end to the oblique distal end of the vascular graft (Figure 1, right). Then the crossclamp on the vascular graft was released, and antegrade flow into the visceral and left renal arteries was reestablished. The right renal artery was subsequently reattached to the prosthesis by means of interposition of a separate, small-diameter $(8 \mathrm{~mm})$ woven vascular graft. The last step comprised a vascular graft-to-aortic bifurcation anastomosis and a final graft-to-graft anastomosis in an end-to-side fashion, thereby completing the repair. Extracorporeal circulation was reestablished for rewarming. Remnants of the aneurysmal wall were loosely wrapped around the aortic graft by using a running suture before the operation was concluded as usual. The patient was extubated 2 days postoperatively, and the further postoperative course was uneventful.

\section{Discussion}

Thoracoabdominal aortic replacement in patients with chronic dissection requires a thorough preparation of one or more vascular patches carrying visceral and renal arteries to be reimplanted into a vascular graft. ${ }^{1,2}$ At first, this implies resection of the dissecting 